\title{
Versatility of Injectable Platelet Rich Fibrin versus autologous blood injection in the Treatment of Chronic Recurrent Temporomandibular Joint Dislocation
}

\author{
Original \\ Article \\ Abdel Aziz Baiomy Abdullah Baiomy', Mohamed Fouad Abdallah Edrees ${ }^{2}$ and \\ Mohammed Mahgob Mohammed Al-Ashmawy ${ }^{1}$ \\ ${ }^{1}$ Department of Oral and Maxillofacial Surgery, ${ }^{2}$ Oral Medicine, Periodontology, \\ Oral Diagnosis and Dental Radiology, Faculty of Dentistry, Al-Azhar University, \\ Assiut, Egypt
}

\begin{abstract}
Purpose: The aim of this study was to investigate the effect of injectable PRF ( i-PRF) versus the autologous blood injection (ABI) in management of chronic recurrent temporomandibular joint (TMJ) dislocation. Patients and Methods: Thirty patients were participated in this study. They were randomly assigned to equalsized groups (group I injected by autologous blood while group II injected by i-PRF). MRIs of the patients were taken preoperatively, one, and three month after the injection. Also, a verbal scale expressing TMJ pain on palpation, maximal mouth opening (MMO), and frequency of luxation were assessed at the same intervals. Results: The results revealed that each treatment appeared to be effective for patients with recurrent TMJ dislocation. Improvement was greater in i-PRF group than those with AB group.

Conclusion: It could be concluded that, each treatment seems to be effective for patients with chronic recurrent TMJ dislocation. Additionally, i-PRF treatment may be highly effective to manage and improve function of chronic recurrent dislocated TMJ compared with ABI .
\end{abstract}

Key Words: Dislocations, Magnetic Resonance Imaging and Temporomandibular Joint.

Received: 02 August 2019, Accepted: 30 August 2019.

Corresponding Author: Abdel Aziz Baiomy Abdullah Baiomy, Department of Oral and Maxillofacial Surgery, Faculty of Dentistry, Al-Azhar University, Assiut, Egypt, Tel.: +20552240879, E-mail: zezobaiomy7@gmail.com.

ISSN: 2090-097X, April 2019, Vol. 10, No. 2

\section{INTRODUCTION}

Dislocation of the condyle of mandible is a common condition that may occur in an acute or chronic form. It is characterized by inability to close the mouth with or without pain. Dislocation can occur in any direction with anterior dislocation being the commonest one. It occurs when one or both mandibular condyles are displaced in front of the articular eminence. It may be reducible when it returns spontaneously to the glenoid cavity, or irreducible when one or two condyles remain dislocated ${ }^{[1,2]}$.

It occurs as a combination of 3 factors; Laxity of mandibular and capsular ligaments, large bony eminence, and spasm of muscle. It may also be due to excessive and irregular movement of the disc condyle complex ahead of articular eminence or due to spasm of temporalis muscle initiated by myotactic reflex ${ }^{[3]}$.

Various factors may lead to dislocation like habitual wide opening of mouth, when yawning ${ }^{[4]}$ or eating, procedures that necessitate prolonged mouth opening like in third molar extraction, endodontic treatment, endotracheal intubation, laryngoscopy ${ }^{[5]}$, transoral fibrotic bronchoscopy ${ }^{[6]}$, steep articular eminence found in skeletal deep bite facial type. Certain medications used for psychiatric problem may also lead to dislocation ${ }^{[7]}$. Some people may be genetically predisposed to joint laxity like in cases of Ehler Danlos syndrome ${ }^{[8]}$.

Various treatment modalities have been described in literature for managing TMJ dislocation including restriction of mandibular range of motion combined with muscle relaxants and soft diet ${ }^{[9]}$, injection of botulinum toxin to various muscles of mastication ${ }^{[10]}$, and injection of sclerosing agents ${ }^{[3]}$. Conservative treatment methods are not always successful and therefore multiple surgical interventions were developed including eminectomy ${ }^{[11]}$, capsular placation $^{[12]}$ temporalis tendon scarification,13 and lateral pterygoid myotomy ${ }^{[14,15]}$.

One of conservative modalities for the treatment of chronic recurrent TMJ dislocation is autohaemotherapy (autologous blood injection $(\mathrm{ABI})$ ) to $\mathrm{TMJ}^{[1,16]}$. It has several advantages such as there is no tissue dissection, postoperative complications such as facial nerve injuries, altered sensation, swelling, infection and pain are all decreased or nonexistent. The procedure can be performed in an office setting with or without sedation under local anesthesia and do not require any sophisticated instrumentation ${ }^{[1]}$. On the other hand, there were some 
concerns about the procedure such as fibrous or bony ankylosis and articular cartilage degeneration.

Recently, Many researchers ${ }^{[17,18]}$, referred the high efficacy of i-PRF in the management of temporomandibular disorders and its safety as autologous material. Also, Miron et $a l .{ }^{[19]}$ demonstrated the ability of i-PRF to release higher concentrations of various growth factors and to induce higher fibroblast migration and expression of PDGF, TGF- $\beta$, and collagen1 thus provide a better environment for the regeneration and repair of the defects. Therefore, i-PRF may be had a promise regenerative effect in chronic dislocated TMJ. Accordingly, current study was atrial to investigate effect of i-PRF versus $\mathrm{ABI}$ in management of recurrent chronic TMJ dislocation.

\section{PATIENTS AND METHODS}

The present study was carried out on 30 patients between the ages of 21 and 49 who had chronic recurrent TMJ dislocation. Seven of the thirty patients had bilateral chronic recurrent TMJ dislocation. All patients were selected based on the clinical and radiographic criteria of TMJ dislocation from those attending the outpatient clinic at Faculty of Dental Medicine, Al-Azhar University, Assiut branch through two years from MAY 2017 to MAY 2019.

All patients participated in this study were complaining of unilateral or bilateral TMJ dislocation for a minimum of 6 months and had previously failed therapy using conservative measures. Preoperative MRI showed there was no articular cartilage degeneration, disc displacement, or osteoarthritis in any patients. It was identified that the patients had unilateral or bilateral condyles anterior to the eminence with their mouths in the open position (Figure $1)$.
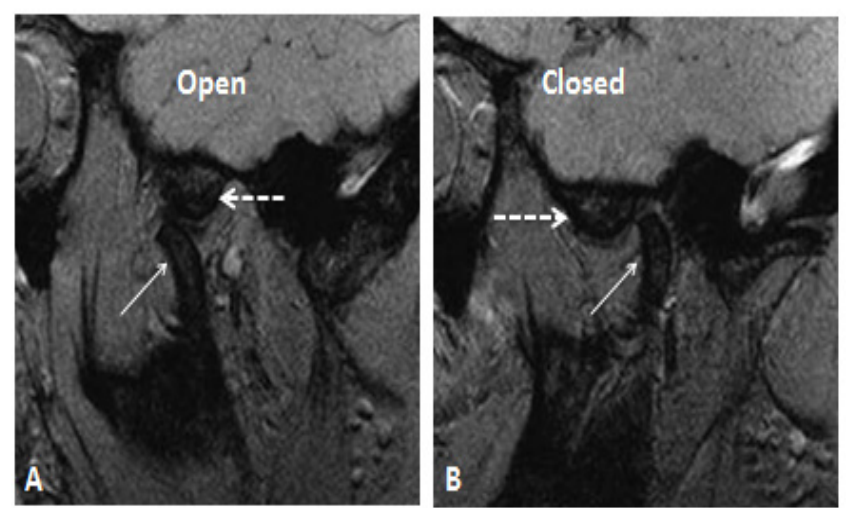

Figure 1: Pre-operative MRI during opened and closed mouth position. Continuous arrows pointed to site of condyle and dashed arrows pointed to articular eminence.

Patients were excluded from the study if they received local steroid injections within 6 months or nonsteroidal antiinflammatory drugs within 1 week prior to randomization. They were also excluded if they had cardiovascular, renal, or hepatic disease, diabetes, anemia, vascular insufficiency, peripheral neuropathy, previous surgery for TMJ, or other
TMJ disorders such as osteoarthritis.

Patients were divided randomly into two equal- sized groups. Each patient was singed an informed written consent having all the details of the procedure. Patients with an odd sequence number were randomly allocated to the ABI group; while the even sequence number patient was automatically placed in i-PRF group.

\section{Preparation of $\boldsymbol{i}$ - PRF ${ }^{[19]}$ :}

In both groups, two tubes of $10 \mathrm{ml}$ of whole blood were withdrawn from the patients' anticubital fossa without anticoagulant. In i-PRF group collected blood were centrifuged at $700 \mathrm{rpm}$ for $3 \mathrm{~min}(60 \times \mathrm{g})$ at room temperature. The upper liquid layer was collected as i-PRF

As part of the single-blind study, blood was also collected from the patients in both groups. All preparation procedures were performed in the clinic without the patient present, by the same investigator. The syringes for both $\mathrm{AB}$ and $\mathrm{i}-\mathrm{PRF}$ were masked with opaque tape to ensure the patient was blinded throughout the trial.

\section{Intra-articular Injection:}

1. Local anesthesia was given to the auriculatemporal nerve.

2. The articular fossa was assumed as located at a point $10 \mathrm{~mm}$ anterior to the tragus of the ear and $2 \mathrm{~mm}$ inferior to the tragal-canthal line (Figure 2).

3. In group AB: $4 \mathrm{~mL}$ blood with a 21- gauge needle was injected in the articular cavity and $1 \mathrm{~mL}$ was injected in the pericapsular tissue. While in group i-PRF $4 \mathrm{~mL}$ i-PRF with a 21- gauge needle was injected in the articular cavity and $1 \mathrm{~mL}$ was injected in the pericapsular tissue.

4. After the completion of the injection, an elastic bandage was applied and left for 24 hours to constrain the joint movements.
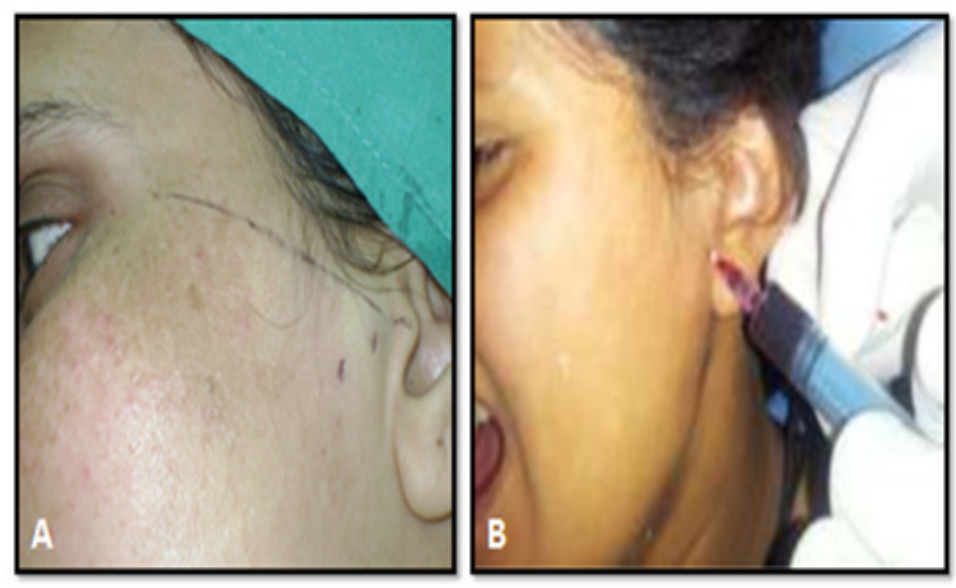

Figure 2: A. Reference line for locating the articular fossa. B. Autologous blood injection. 


\section{Postoperative evaluation:}

Clinical evaluation: The patients subjectively assessed their TMJ pain intensity on palpation using the verbal analog scale (VAS; scale 0 - 10), and the maximal interincisal mouth opening (MMO) was clinically measured in millimeters. Moreover, frequency of luxations (number of locking episodes per month) was assessed just before the injection procedure, one, and three months after the injection. The collected data were then statistically analyzed.

\section{Radiographic evaluation:}

All of the patients were scanned by MRI before injection, one month, and 3 months after injection to assess position of condyle and articular disk. MRI was carried out with a 1.5T, MR scanner (Gyroscan Intera Master; Philips Healthcare, Eindhoven, The Netherlands) and a dedicated, circular polarized transmit and receive TMJ coil. The MRI protocol included bilateral sagittal oblique proton density images of the right and left sides in both the closed mouth (maximum intercuspation) and maximum open mouth positions. The data were collected on a $240 \times 167$ matrix, with a field of view of $15 \mathrm{~cm}$ giving a pixel size of $0.625 \times 0.9 \mathrm{~mm}$. With the patient in a supine position, 12 parasagittal slices were obtained for each TMJ by using a turbo spin-echo proton density sequence (TR of $1500 \mathrm{~ms}$, echo time of $30 \mathrm{~ms}$ ), number of excitations was 4.00 with $3 \mathrm{~mm}$ slice thickness. MR images were corrected to the horizontal angulation of the long axis of the condyle.
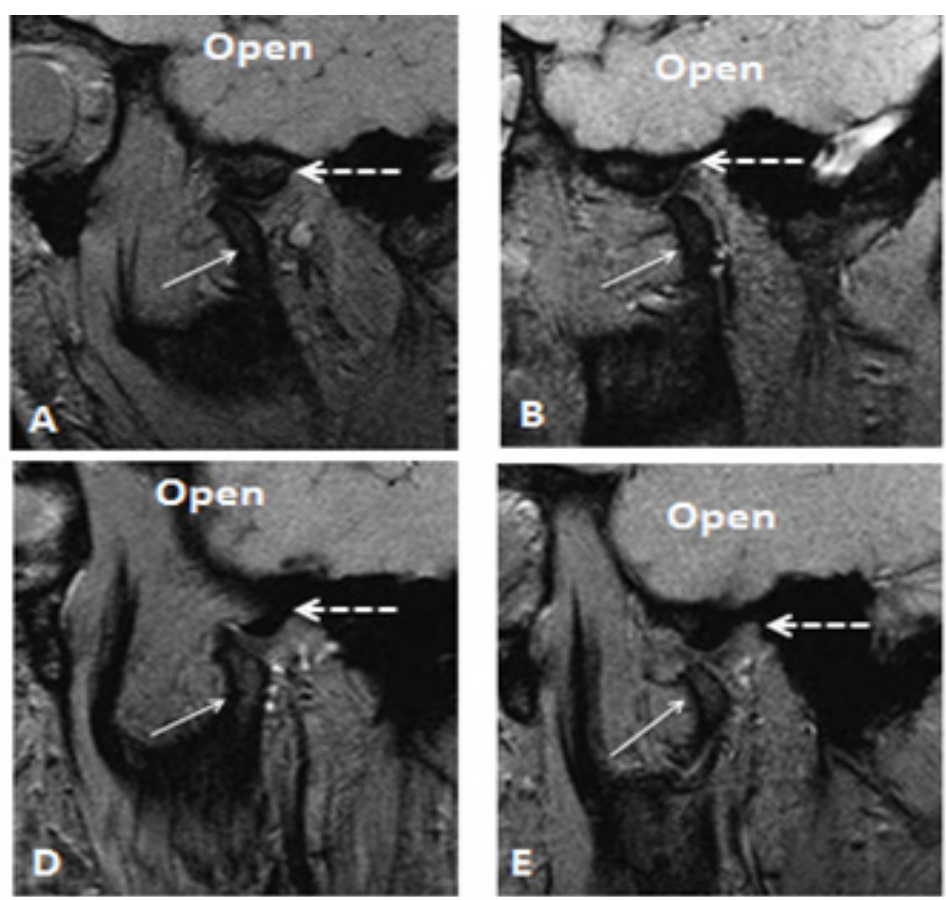

\section{Statistical analysis:}

Differences and percentage of change between particular groups were analyzed by paired Student's T-test. Statistical analysis was performed by software program, SPSS 20 ® (Statistical Package for Social Sciences) at level of significance $P \leq 0.05$.

\section{RESULTS}

All the patients tolerated the procedure well. There were no infections or any other complications in any of the patients. The post- operative pain was tolerable in all the patients and last for only few days after the procedure. At end of the study in group I, two patients showed recurrent dislocation while in group II, none patients had TMJ dislocation.

\section{Radiographic results:}

Except two patients in $\mathrm{AB}$ group, postoperative MRIs of all patients (taken with the open - mouth position) at 3 months showed that no difference between both groups in radiographic presentation. Where, they revealed that the condyle was either at the apex of the eminence or posterior to it. Although, postoperative MRIs of all patients at one month presented that early reduction of condyle with articular disc to normal position during open mouth position in i-PRF group than $\mathrm{AB}$ injection group.

Moreover, there was no structural variation that described the prevention of the dislocations such as fibrosis in MRI findings. Moreover, Hematoma due to injection was disappeared without any alteration of the joint tissues.

Figure 3: MRI images of the left TMJ in both groups. A. Open-mouth position before injection in group i-PRF. B. Open -mouth position after injection in group i-PRF at one month. C. Open -mouth position after injection in group i-PRF at 3 months D. Openmouth position before $\mathrm{AB}$ injection $\mathbf{E}$. Open mouth position after $\mathrm{AB}$ injection at one month. F. Open mouth position after $\mathrm{AB}$ injection at 3 months. Continuous arrows pointed to site of condyle and dashed arrows pointed to articular eminence. 


\section{Statistical results:}

\section{Interincisal mouth opening:}

The overall average interincisal mouth openings of groups were shown in table 1. The overall average interincisal mouth openings before treatment was $55.8+1.59$ in $\mathrm{AB}$ group and $56.03+0.72$ in $\mathrm{i}-\mathrm{PRF}$ group. During the study in the $\mathrm{AB}$ group range of interincisal mouth opening is decreased from $55.8+1.5 \mathrm{~mm}$ at baseline to $39.4+1.98 \mathrm{~mm}$ at one month till reach to $49.4+1.82 \mathrm{~mm}$ at 3 months follow up after injection. In i-PRF group, range of interincisal mouth opening decreased from $56.03+0.72 \mathrm{~mm}$ at baseline to $40.6+1.53 \mathrm{~mm}$ at one month and to $45.2+1.07 \mathrm{~mm}$ at 3 months follow up after injection. Regarding relative improvement in the range of interincisal mouth opening, groups of the study showed decrease in of interincisal mouth opening till reach to normal measurement of interincisal mouth opening at last day of the study without any significant difference between both groups except at last day of study. Hence, there was high significant difference between both groups at last day of study.

\section{Pain intensity:}

Table 1 presented that in the $\mathrm{AB}$ group ,the main pain subscale scores were $8.8+0.91$ at baseline and reach to 6.5 +1.67 (26.14\% improvement) at one month and $2.7+1.33$
(72.9\% improvement) at last day of the study. While in the i-PRF group, the main pain subscale scores were $8.9+1.11$ at baseline and reach to $4.63+0.55$ (8.87\% improvement) at 4 weeks and $1.1+0.95$ (84.71\% improvement) at last day of the study. Both groups showed significant improvements (decrease of pain intensity) although i-PRF group showed early improvement at all intervals with higher percent than AB group.

\section{Frequency of luxations:}

The main frequency of luxation subscale scores of $\mathrm{AB}$ group were $9.5+2.01$ at baseline, $6.8+1.47$ (28.42\% improvement) at first month from the study and $1.2+0.78(86.31 \%$ improvement $)$ at the last day of the study. While in the i-PRF group, the main frequency of luxation subscale scores were $9.4+2.06$ at baseline, $6.8+1.68(27.65 \%$ improvement $)$ at the first month and $0.1+0.63(93.86 \%$ improvement $)$ at the last day of the study.

Regarding to relative improvement in the frequency of luxation (decrease number of luxation of TMJ), both groups showed significant improvements at all intervals of study. Although, there were highly significant difference between both groups at last day of study. This was presented in the percentages of the improvement which higher in i-PRF group than $\mathrm{AB}$ group at last day of the study.

Table 1: Means and standard deviations of the Interincisal mouth openings measurements, VAS scores, and luxation frequencies per month and test of significances between $\mathrm{AB}$ and i-PRF groups at different times

\begin{tabular}{|c|c|c|c|c|c|c|c|c|c|}
\hline Group & Prepreoperative & 1 Month & 3 Months & Preoperative & 1 Month & 3 Months & Preoperative & 1 Month & 3 Months \\
\hline & \multicolumn{3}{|c|}{ Interincisal Mouth Opening } & \multicolumn{3}{|c|}{ VAS Scores } & \multicolumn{3}{|c|}{ luxation Frequencies } \\
\hline $\mathrm{AB}$ & $55.8+1.59$ & $39.4+1.98$ & $49.4+1.82$ & $8.8+0.91$ & $6.5+1.67$ & $2.7+1.33$ & $9.5+2.01$ & $6.8+1.47$ & $1.2+0.78$ \\
\hline i-PRF & $56.03+0.72$ & $40.6+1.53$ & $45.2+1.07$ & $8.9+1.11$ & $4.63+0.55$ & $1.1+0.95$ & $9.4+2.06$ & $5.8+1.68$ & $0.1+0.63$ \\
\hline t-value & $0.262-$ & -1.429 & -3.352 & 0.088 & 3.612 & 1.358 & .096 & 0.000 & 0.000 \\
\hline Sig. & 0.799 & 0.187 & $0.008^{*}$ & 0.932 & $0.006^{*}$ & $0.048^{*}$ & 0.925 & 1.000 & 1.000 \\
\hline
\end{tabular}

* Significant at $P \leq 0.05$ Highly significant at $P \leq 0.001$.

\section{DISCUSSION}

Condyle dislocation (or hypermobility) of TMJ is one of the most frequent TMJ disorders in humans ${ }^{[20]}$. In the case of hypermobility, the condyle reaches a position in front of the articular tubercle at wide mouth opening, which can be caused by abnormalities in the shape of the joints, by ligament looseness or by reduced muscle tension ${ }^{[2]}$.

Various nonsurgical and surgical modalities have been described in the literature with variable success. There is no definite consensus regarding superiority of any treatment modality over the other ${ }^{[10-15]}$. Although, there are many clinical studies about a high success rate of autologous blood injection into $\mathrm{TMJ}^{[1,2]}$. The procedure is easy to perform and it causes no foreign body reaction. There have been some successful clinical studies in the literatures ${ }^{[16]}$.
In the same side, the prevalence of the use of autologous blood products in TMJ disorders treatment has been increasing. Hence, many researchers referred the high efficacy of platelet-rich plasma in the management of temporomandibular disorders and its safety as autologous materia $^{[121,22]}$. One of the drawbacks of PRP is the additional use of anti-coagulants, known to delay wound healing. Further, a second-generation platelet concentrates termed platelet rich fibrin (PRF) was developed to improve wound healing in comparison to PRP. Standard PRF contains a 3-dimensional fibrin matrix following centrifugation, however this is not ideal for injections as it is cumbersome to handle. A pioneer development of the low speed centrifugation method introduced the concept of injectable PRF (i-PRF), a liquid formulation of PRF without using anticoagulants $^{[1,, 23]}$. 
In the same context, Amirthraj ${ }^{[18]}$ assessed i-PRF injection effect in degenerative TMJ disease and concluded that i-PRF when injected reduces inflammation, provide pain relief, improve function and stimulate possible cartilage regeneration at the site of injury. However, none of these reports dealt with recurrent TMJ dislocation. Moreover, no trial has directly compared the effectiveness of i-PRF and $\mathrm{AB}$ injections in recurrent $\mathrm{TMJ}$ dislocation.

Our study compared the clinical and MRIs outcomes of each modality for the treatment of recurrent TMJ dislocation. This study was focused on the potential benefits of i-PRF treatment on recurrent TMJ dislocation in comparison with $\mathrm{AB}$; i-PRF treatment resulted in earlier pain relief and decrease of luxation frequency than $A B$ treatment. These results matched with MRIs findings that proved effectiveness of i-PRF more than $\mathrm{AB}$ injection in recurrent TMJ dislocation.

The results of this study appeared to show the beneficial effects of both $\mathrm{AB}$ and $\mathrm{i}-\mathrm{PRF}$ injection therapies in patients with recurrent TMJ dislocation, with improvements in both pain relief and function. The improvements (relief of pain) in the mean pain intensity total scores were 48.87 $\%$ at one month and resulting in $83.71 \%$ improvement at 3 months after last injection in i-PRF group. On the other hand, the improvement percentages in the mean pain intensity total scores were $26.14 \%$ at one month, $74.9 \%$ improvement at 3 months after last injection in the DP group. Regarding to frequency of luxation, both groups showed high percentages of improvement (decrease number of luxation) at last day of the study $86.31 \%$ in the $\mathrm{AB}$ group and $93.86 \%$ in the i-PRF group with highly significant difference between both groups at last day of study. These results may be explained by direct action of $\mathrm{i}-\mathrm{PRF}$ on tissue is rapid than indirect action of $\mathrm{AB}$ injection take time till inflammatory response and healing process was occurred. This explanation goes parallel with studies of Amirthraj ${ }^{[18]}$, and Miron et al. ${ }^{[19]}$ that demonstrated the ability of i-PRF to release higher concentrations of various growth factors and to induce higher fibroblast migration and thus provide a better environment for the regeneration and repair of injured tissue.

In the same direction, present study measurements of interincisal mouth openings were reduced at one month interval then increased gradually till reach to normal mouth opening at 3 months with high significant difference between both groups at 3 months intervals. This may be explained by inflammatory response at injection site lead to reduced mouth opening. Really, results of the present study were matched with several clinical studies on $A B$ such as Machon et al. ${ }^{[1]}$ and Hasson et al. ${ }^{[2]}$ studies.

In the current study, MRIs results at 3 months showed that no difference between both groups in radiographic presentation. Where, they revealed that the condyle was either at the apex of the eminence or posterior to it. Also, there was no structural variation that described the prevention of the dislocations such as fibrosis in MRI findings. These results of treatment were in agreement with the previous studies as mentioned above. However, contrary to the Roosendaal et al. ${ }^{[24]}$, and Lafeber et al. ${ }^{[25]}$ studies which claimed that the contact of cartilage with blood caused the changes in chondrocyte metabolism and thus cartilage destruction.

In conclusion, both of $\mathrm{AB}$ and $\mathrm{i}-\mathrm{PRF}$ were safe and simple treatment modalities for recurrent TMJ dislocation. However, i-PRF was more potentially effective treatment for recurrent $\mathrm{TMJ}$ dislocation than $\mathrm{AB}$ injection. Moreover, i-PRF injection in TMJ led to earlier reduction of pain and improvement of function than $\mathrm{AB}$ injection.

\section{CONFLICT OF INTEREST}

There are no conflicts of interest.

\section{REFERENCES}

1. Machon V, Abramowicz S, Paska J, Dolwick M. Autologous blood injection for the treatment of chronic recurrent temporomandibular joint dislocation. J Oral Maxillofac Surg 2009; 67: 114-9.

2. Hasson $\mathrm{O}$, Nahlieli O. Autologous blood injection for treatment of recurrent temporomandibular joint dislocation. Oral Surg Oral Med Oral Pathol Oral Radiol Endod 2001; 92: 390- 3.

3. Shorey C, Campbell J. Dislocation of the temporomandibular joint. Oral Surg Oral Med Oral Pathol Oral Radiol Endod. 2000; 89 (6): 662-8.

4. Avidan A. Dislocation of the temporomandibular joint due to forceful yawning during induction with propofol. J Clin Anesth. 2002;14(2):159- 60.

5. Rattan V, Arora S. Prolonged temporomandibular joint dislocation in an unconscious patient after airway manipulation. Anesth Analg. 2006; 102 (4): 1294.

6. Durga V, Millns J, Smith J, Manoeuvers used to clean the airway during fibreoptic intubation. $\mathrm{Br} \mathrm{J}$ Anaesth. 2001; 87 (2): 207-11.

7. Undt G, Weichselbraun A, Wagner A, Kermer C, Rasse M. Recurrent mandibular dislocation under neuroleptic drug therapy, treated by bilateral eminectomy. J Craniomaxillofac Surg. 1996; 24 (3): 184-8.

8. Poveda-Roda R, Bagán J, Díaz-Fernández J, Hernández-Bazán S, Jiménez-Soriano $\mathrm{Y}$. Part I Review of temporomandibular joint 
pathology. Part I: Classification, epidemiology and risk factors. Med Oral Patol Oral Cir Bucal. 2007; 12 (4): 292-8.

9. Martinez-Perez D, Garcia Ruiz-Espiga P. Recurrent temporomandibular joint dislocation treated with botulinum toxin: Report of 3 cases. J Oral Maxillofacial Surg. 2004; 62 (2): 244-6.

10. Myrhaug H. A new method of operation for habitual dislocation of the mandible: review of former methods of treatment. Acta Odontol. 1951; 9 (3-4): 247-60.

11. MacFarlane W. Recurrent dislocation of the mandible: Treatment of seven cases by a simple surgical method. Br J Oral Maxillofac Surg. 2004; 14(3): 227-9.

12. Gould J. Shortening of the temporalis tendon for hypermobility of the temporomandibular joint. $\mathrm{J}$ Oral Surg. 1978; 36 (10): 781-3.

13. Sindet-Pedersen S. Intraoral myotomy of the lateral pterygoid muscle for treatment of recurrent dislocation of the mandibular condyle. J Oral Maxillofac Surg. 1988; 46 (6): 445-9.

14. Miller G, Murphy E. External pterygoid myotomy for recurrent mandibular dislocation: Review of the literature and report of a case. Oral Surg Oral Med Oral Pathol. 1976; 42 (6): 705-16.

15. Kato T, Shimoyama T, Nasu D Kaneko T, Horie N, Kudo I. Autologous blood injection into the articular cavity for the treatment of recurrent temporomandibular joint dislocation: A case report. J Oral Sci 2007; 49 (3): 237-9.

16. Albilia J , Herrera-Vizcaino C, Weisleder H, Choukroun J, Ghanaati S . Liquid platelet-rich fibrin injections as a treatment adjunct for painful temporomandibular joints: preliminary results. Cranio. 2018, 20: 1-13.

17. Amirthraj A. The Clinical Efficacy of Injectable Platelet Rich Fibrin in the Treatment of Degenerative Temporomandibular Joint Disease . Nov Tech Arthritis Bone Res. 2018; 3 (2): 1-2.

18. Miron RJ, Fujioka-kobayashi M, Hernandez M . Injectable platelet rich fibrin (i-PRF): opportunities in regenerative dentistry? Clin Oral Investig 2017, 21 (8): 2619-27.

19. Antczak-Bouckoms A. Epidemiology of research for temporomandibular disorders. J Orofac Pain 1995; 9: 226-34.

20. Sharma S, Thakar D .The Clinical Efficacy of Autologous. Concentrated Platelets in Treatment of Tmj Disorders - a pilot study. 2014, 4 (3): 70-74..

21. Kon E, Mandelbaum B, Buda R, et al. Platelet-rich plasma intra-articular injection versus hyaluronic acid viscosupplementation as treatments for cartilage pathology: from early degeneration to osteoarthritis. J Arthroscopy 2011; 27: 1490-501.

22. Choukroun J, Ghanaati S. Reduction of relative centrifugation force within injectable plateletrich-fibrin (PRF) concentrates advances patients' own inflammatory cells, platelets and growth factors: the first introduction to the low speed centrifugation concept. Eur J Trauma Emerg Surg 2018, 44 (1): 87-95.

23. Roosendaal G, Lafeber F. Blood-induced joint damage in hemophilia. Semin Thromb Hemost 2003; 29: 37-42.

24. Lafeber F, Miossec P, Valentino L. Physiopathology of haemophilic arthropathy. Haemophilia 2008; 14 (4): 3-9. 